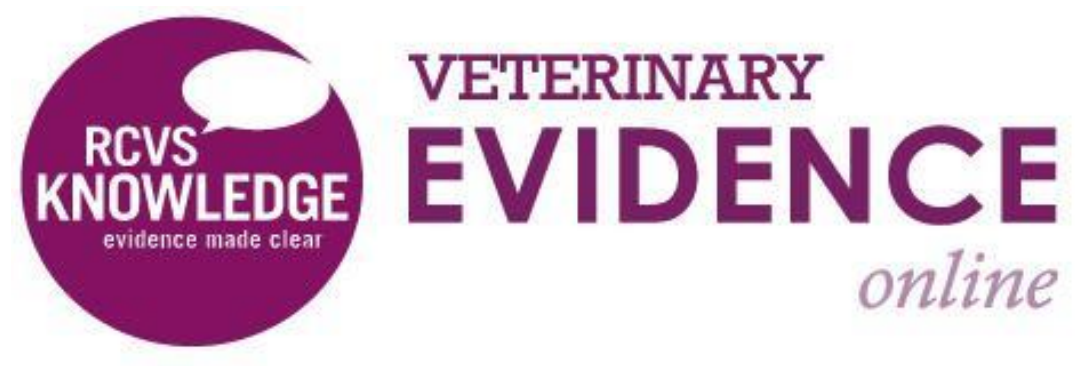

\title{
Use of Intravenous Lipid Emulsion in Dogs With Suspected Tremorgenic Mycotoxicosis: 53 Cases
}

Foteini Kormpou DVM, PGcert(CVP) MRCVS ${ }^{1 *}$

Aoife O'Sullivan MVB CertAVP(ECC) MRCVS ${ }^{2}$ Laura Troth BVMedSci BVM BVS MRCVS ${ }^{2}$ Sophie Adamantos BVSc CertVA DACVECC DipECVECC MRCVS FHEA ${ }^{1}$

\footnotetext{
${ }^{1}$ Langford Vets, University of Bristol, Langford, BS405DU

${ }^{2}$ Vets Now, Penguin House, Dunfermline, KY11 8SG

* Corresponding Author (fwteini2008@hotmail.com)
}

ISSN: 2396-9776

Published: 24 May 2018

in: Vol 3, Issue 2

DOI: http://dx.doi.org/10.18849/ve.v3i2.166

Reviewed by: Tiffany Blackett (BvetMed MRCVS) and Nicola Bates (BSc (Brunel), BSc (Open), MSc, MA, SRCS) 
Objective: The purpose of this study is to report the use of intravenous lipid emulsion (ILE) in dogs presenting with suspected tremorgenic mycotoxicosis based on signs of intoxication and known ingestion of mouldy food or compost.

Background: ILE is being used for toxicoses associated with fat soluble agents. As tremorgenic mycotoxins are lipophilic in nature, the use of ILE may be of benefit in these patients.

Methods: Medical records of all dogs examined at any Vets Now clinic between November 2012 and November 2016, in which ILE had been used, were reviewed and 53 cases were included in the study.

Results: In $96 \%$ of cases there was clinical improvement recorded post ILE administration within a median time of 4 hours. No adverse effects were seen during the infusion or up to the time of discharge.

Conclusion: The administration of ILE seems to be associated with a beneficial effect in the short term in dogs. Prospective evaluation of this therapy is warranted to further evaluate the use of ILE in these cases.

INTRODUCTION

Tremorgenic mycotoxicosis is a common intoxication in dogs, due to their indiscriminate eating habits. A presumptive diagnosis is made in most cases based upon history and clinical findings. Known ingestion of, or possible access to, mouldy food, garbage, or compost is highly suggestive (Barker et al., 2013). Signs usually occur within 30 minutes of ingestion, although rarely, can be delayed for several hours (Talcott, 2013). The most common signs of tremorgenic mycotoxin exposure reported to the Veterinary Poisons Information Service (VPIS) include ataxia, tremors, restlessness, hyperaesthesia, tachycardia and vomiting. In severe cases opisthotonus, seizures and coma can be seen. Signs can sometimes progress to hyperthermia, exhaustion, rhabdomyolysis, dehydration and hypoglycaemia. Clinical signs usually resolve gradually within 24-96 hours. Approximately 20 mycotoxins have been identified as tremorgens. Those most frequently reported to affect dogs are penitrem A and roquefortine C, which are produced by Penicillium spp (Eriksen et al., 2010). After ingestion, the tremorgenic mycotoxins present in contaminated food are thought to cross the blood-brain barrier due to lipophilic properties (Schell, 2000). Multiple mechanisms of action have been suggested for tremorgenic mycotoxins. Rats injected with penitrem A show marked increases in the release of gammaaminobutyric acid GABA, glutamate, and aspartate at cerebrocortical synapses (Norris et al., 1980). A similar interference with neurotransmission within the cerebellum is thought to be the reason behind the characteristic tremor in dogs (Eriksen et al., 2010); however, it is generallybelieved that the mechanism of action differs for each toxin involved in the exposure. Further researchis warranted to identify specific mechanisms of action.

Treatment is symptomatic and supportive and usually involves gastrointestinal decontamination, management of neurological signs with muscle relaxants,anti-seizure drugs and management for hyperthermia as required. Due to the rapid absorption of mycotoxins and rapid onset of clinical signs, the window of opportunity to safely induce emesis is generally limited (Boysen et al., 2002; Crandell, 2006). Therefore, once clinical signs are appropriately controlled, gastric lavage should be considered in these cases. Activated charcoal with or without a cathartic may then be instilled through the gastric tube before it is removed to reduce toxin 
exposure. There is evidence that the common mycotoxins are excreted in bile, suggesting the likelihood of hepatic recirculation (Puschner et al., 2009) and the need for repeated doses of activated charcoal over 2 to 3 days.

ILE was initially used to treat local anaesthetic overdoses in people. In recent years, reports of the use of ILE in veterinary medicine has increased (Fernandez et al., 2011; Epstein et al., 2013; Bates et al., 2013) and is advocated by the VPIS and the Animal Poison Control Center (APCC) for toxicoses associated with fatsoluble agents.

The exact mechanism of action for ILE has yet to be determined. The "lipid sink" theory has been proposed as a possible mechanism as well as direct cardiovascular effects (Fettiplace et al., 2015; Rothschild L et al. 2010). During infusion of ILE, a lipid phase in the aqueous portion of the blood is created, acting as a sink for lipophilic drugs, preferentially pulling them out, or keeping them out, of the tissues (Fettiplace et al., 2015). Its direct cardiovascular effect involves providing a source of energy to the myocardial cells. The use of ILE for the treatment of lipophilic drug toxicity is increasing. French et al., 2001, suggested that lipid soluble toxicants with a partition coefficient (LogP) greater than 1 or 2 and a high volume of distribution (Vd) will respond better to ILE therapy. However, ILE's efficacy in non-local anaesthetic toxicity appears to be heterogenous and the quality of evidence to support its use in these cases remains low (Levine et al., 2016). The authors are aware of anecdotal reports of itsuse in mycotoxicosis, however, there is only one published case report of the use of ILE in dogs with tremorgenic mycotoxin intoxication (Parratt, 2014). In this report, although the dog improved with lipid, it was not sufficient to withdraw additional treatment. Roquefortine was confirmed in vomitus in this case.

The purpose of this study is to report the use of ILE in dogs presenting with suspected tremorgenic mycotoxicosis, based on signs of intoxication and known ingestion of mouldy food or compost; with the hypotheses that, as tremorgenic mycotoxins are lipophilic in nature(Puschner, 2009), the use of ILE may be beneficial in these patients.

\section{RESULTS}

Fifty-three dogs met the criteria for inclusion in the study. Dogs were a median of 3.8 years old (range 0.5-13). There were 28 males and 26 females. Common breeds represented included Labrador retrievers $(n=7)$ and beagles (7). Nineteen other breeds were represented by less than 4 dogs each. There were 7 mixed-breed dogs.

Historical complaints and clinical abnormalities at the time of presentation included tremors $(n=48)$, ataxia $(n=17)$, vomiting $(n=15)$, hyperthermia $(n=15)$, seizures $(n=13)$, hyperaesthesia $(n=8)$, tachycardia $(n=7)$, diarrhoea $(n=4)$, collapse $(n=2)$, abdominal pain $(n=1)$. All dogs were presented primarily for neurological signs.

Duration of signs prior to presentation was not consistently recorded in the medical records, but for the $25 / 53$ cases that the duration had been recorded it was a median of 2 hours (range 16 minutes to 8 hours).

Treatment varied between individuals. ILE was used for all the cases included in this study, but never as the sole agent. The exact dose and rate of ILE administered was recorded in 46 dogs (87\%). In 34/46 (73\%) cases an intravenous bolus of $1.5 \mathrm{ml} / \mathrm{kg}$ of a $20 \%$ solution of intralipid followed by $0.25-0.5 \mathrm{ml} / \mathrm{kg} / \mathrm{min}$ constant rate infusion for 30-60 minutes was administered. The remaining 12 cases had a variety of doses with 7 treated with a bolus followed by infusion, 3 treated with a bolus only and 2 treated with an infusion only. Seven cases (15\%) had one repeated dose and four $(9 \%)$ had two repeated doses. The repeated doses ranged from 
$0.25 \mathrm{ml} / \mathrm{kg}$ to $1.5 \mathrm{ml} / \mathrm{kg}$ and were always the same as the initial bolus dose that was administered in each case. Benzodiazepines (diazepam or midazolam) were administered in 38/53 (71\%) cases, intravenous fluid therapy in $31 / 53(58 \%)$ cases, methocarbamol in $22 / 53(42 \%)$ cases, activated charcoal in $21 / 53(40 \%)$ cases, phenobarbital in $10 / 53(19 \%)$ cases, propofol in $10 / 53(19 \%)$ cases, maropitant in $5 / 53$ (9\%) cases, medetomidine in $5 / 53(9 \%)$ cases, apomorphine in $4 / 53$ (8\%) cases, opioids in $4 / 53(8 \%)$ cases, glucose in $2 / 53$ (4\%) cases, mannitol in $1 / 53(2 \%)$ case and co-amoxiclav in $1 / 53(2 \%)$ case. The timing that ILE was given in relation to the timing of other drugs or intravenous fluid therapy ranged and was not consistently recorded. In most cases where diazepam was used, this was the first drug to be administered. However, it appeared that diazepam alone was never sufficient to control the signs and, as a result, administration of additional drugs was deemed necessary. This agrees with previous reports suggesting that diazepam alone is not usually effective in mycotoxicosis cases (Boysen et al., 2002; Young et al., 2003).

Hospitalisation time at the clinic was a median of 12 hours (range 3-72). In 51/53 (96\%) cases there was clinical improvement recorded in the clinical notes following ILE. The time that clinical signs took to improve or resolve after ILE was a median of 4 hours (range 30 minutes to 36 hours). In 23/53 (43\%) there was complete resolution of the signs; 12 of these cases were discharged to their primary care vets and 11 of them were discharged home. From the cases with complete resolution of signs, five had a repeated dose of ILE.

In total, 19 dogs (36\%) were discharged home, 33 dogs (62\%) were transferred back to the primary care vet and one dog was euthanased due to the lack of improvement and financial restrictions. In one dog, no clinical information was recorded in the clinical records after administration of ILE. This dog was discharged to its primary care vet. For the 33 dogs that were discharged to the primary care vet, no follow up information is available.

\section{DISCUSSION}

The results of this study suggest that the median time to clinical improvement in dogs with suspected tremorgenic mycotoxicosis treated with ILE is 4 hours. This compares favourably with previous reports which suggest that signs resolve completely within $24-96$ hours (Barker et al., 2013). There is limited information about the natural progression of this disease, and clinical experience suggests that signs improve over variable periods of time. The improvement seen in these cases may have been natural progression of disease. The prognosis for dogs treated for tremorgenic mycotoxins is reportedly excellent and this was confirmed in this study with only one dog being euthanased. The administration of ILE seems to be associated with a beneficial effect in the short term in this population of animals; it appears that treatment with lipid resulted in reduced recovery time in dogs with tremorgenic mycotoxicosis, compared to expected recovery time based on literature reports (Barker et al., 2013; Young et al., 2003). No adverse effects were recorded during the infusion or up to the time of discharge.

There are a number of considerations that need to be taken into account for this study. Due to the retrospective nature of the study some of the data were not consistently recorded in the medical records. Importantly long-term follow-up was not available, however all dogs, except for one, were considered to have improved and were clinically stable at the time of discharge. The timing ofthe ILE administration in relationtothe timing ofthe other drugs or intravenous fluid therapy ranged and was not always recorded; hence it would be hard to confirm that it was solely the ILE that resulted in the clinical improvement in these cases. Further to this, interactions between ILE and standard treatments are not currently known, but it is likely that any lipophilic drug, including diazepam, phenobarbital and propofol, will have minimal action during infusion of ILE.

The dosage of ILE used varied between the cases and was not always recorded. However, in most cases standard protocols were followed and included an intravenous bolus of $1.5 \mathrm{ml} / \mathrm{kg}$ of a $20 \%$ ILE, followed by a constant rate infusion CRI of $0.25-0.5 \mathrm{ml} / \mathrm{kg} / \mathrm{min}$ for $30-60$ minutes, which was repeated as necessary. 
Reported dose ranges in the literature include boluses of $1.5 \mathrm{ml} / \mathrm{kg}-2.0 \mathrm{ml} / \mathrm{kg}$ over 2-15 minutes, then as a CRI $0.06 \mathrm{ml} / \mathrm{kg} / \mathrm{min}$ to $0.5 \mathrm{ml} / \mathrm{kg} / \mathrm{min}$ for multiple hours (Gwaltney-Brant, 2012). No maximum daily dose has been determined in veterinary patients (Kuo et al., 2013).

Methocarbamol was used in $40 \%$ of cases to control the tremors. The beneficial use of methocarbamol in suspected mycotoxicosis cases has been reported previously (Schell, 2010). As tremors may last for several days, oral doses of methocarbamol may be continued after the dog is discharged from the hospital. Onset of its action is reportedly 30 minutes and its peak effect is 1-2 hours post oral administration. It is possible that the improvement seen in some of the dogs was due solely to methocarbamol administration.

Finally, the diagnosis of mycotoxicosis in these cases was presumptive and was based on the history and clinical signs. Definitive diagnosis is difficult and not commonly reached. This is mainly due to the fact that the results cannot be obtainable in a clinically relevant timeframe and that tremorgenic mycotoxicosis is always treated empirically. Definitive diagnosis involves analysis of vomitus or stomach contents, blood, urine, or the suspected contaminated material. Analytic methods include identification of the mould through culture of the organism or detection of the toxins themselves (Eriksen et al. 2010; Rundberget et al., 2002; Young et al.,

2003). Liquid chromatography has also been used to screen for the specific toxins penitrem $A$ and roquefortine in serum (Puschner, 2009) and urine samples (Tiwary, 2009). These tests are available from some specialised diagnostic laboratories. On necropsy, penitrem A has been isolated from the liver, kidney, and brain (Eriksen et al., 2010).

Even though no acute adverse effects of this therapy were reported in any of the dogs included in this study, these have been rarely reported. Potential complications that have not been reported in dogs include sepsis secondary to bacterial contamination and thrombophlebitis (Kirsten et al., 2012). In people, lipaemia, hepatosplenomegaly, jaundice, haemolysis, coagulopathy, fat emboli, and thrombocytopenia are potential concerns (Kirsten et al., 2012). In addition, hypersensitivity reactions and possible interference with other drugs can be seen (Gwaltney-Brant, 2012). In a case series, one dog had swelling and pain associated with extravasation of the ILE (Bates et al., 2013).There is a single case report of a cat which presented with persistent gross lipaemia and suspected corneal lipidosis following treatment for ivermectin toxicity with ILE (Seitz et al., 2016). Since there are no current studies evaluating the safety of ILE use in acute toxicities (Kuo et al., 2013), owners should be informed of its off-label use (Haworth et al., 2012).

The results of this study indicate that ILE may be a valuable addition to the clinician's treatment options for suspected exposure to tremorgenic mycotoxins. Prospective evaluation of this therapy is warranted to clarify whether clinical signs are more rapidly resolved through the use of ILE versus standard therapies. The use of a scoring system to quantify severity of signs such as that reported for use in cats with permethrin toxicity by Peacock et al., 2015, would be useful to allow comparison of therapies as well as evaluating the clinical course of disease. Further research needs to be conducted in a standardised manner in order to determine specifics for ILE dosing, most effective time period for administration, length of administration, and which patients are most likely to respond. As this is a toxicity that is most commonly seen in first opinion practice creating methods by which randomised clinical trials are easily performed is required.

\section{METHODS \& MATERIALS}

\section{Criteria for Selection of Cases}

Medical records of all dogs treatedat a Vets Now (out-of-hours emergency-care service provider) clinic between November 2012 and November 2016, in which ILE had been used, were reviewed. Cases were eligible for inclusion in the study if the dog had been treated with ILE and had a highly suspicious diagnosis of mycotoxicosis from the history and the physical examination findings. Cases were considered to be highly suspicious for mycotoxicosis if the following criteria were met: peracute onset of neurological signs with or without gastrointestinal signs AND known ingestion of mouldy food/ compost according to the owner. For the 
purposes of this study, when duration of signs prior to presentation is recorded as "few hours", this is considered to be less than 6 hours.

\section{Procedures}

Data obtained from the medical records included age, breed, presenting signs, duration of signs prior to presentation, treatment, progress during hospitalisation, hospitalisation time and outcome at the time of discharge (dead, alive and discharged home or alive and discharged to primary care veterinary practice). Due to the nature of out-of-hours veterinary care in the UK, long-term follow-up information was not available, since all dogs were discharged either to the owners careor to their primary care veterinary practice by the end of the shift, if they were considered to be stable enough to travel.

Data was entered into a spreadsheet and cases were evaluated by two of the authors (FK and LT) Data were extracted into a Statistical Package (SPSS v 23.0). Data are presented descriptively.

CONFLICT OF INTEREST

The authors declare no conflicts of interest.

REFERENCES

1. Barker, A.K. et al. (2013). Tremorgenic Mycotoxicosis in dogs. CompendContinEduc Vet 35(2):E2.

2. Bates, N. et al. (2013). Lipid infusion in the management of poisoning: a report of 6 canine cases. Veterinary Record 172, 339. DOI: http://dx.doi.org/10.1136/vr.101036

3. Boysen, S., Rozanski, E., Chan, D., Grobe, T., Fallon, M. and Rush, J. (2002). Tremorgenic mycotoxicosis in four dogs from a single household. Journal of the American Veterinary Medical Association, 221(10), pp.1441-1444. http://dx.doi.org/10.2460/javma.2002.221.1441

4. Crandell D. Toxicological emergencies. In: Mathews KA, ed. Veterinary Emergency and CriticalCare Manual. 2nd ed. Guelph: Lifelearn Publishers; 2006:630-640.

5. Epstein, S.E. and Hollingsworth, S.R. (2013). Ivermectin-induced blindness treated with intravenous lipid therapy in a dog. Journal of Veterinary and Emergency Critical Care 23, 58-62. DOI: http://dx.doi.org/10.1111/vec.12016

6. Eriksen, G.S. et al. (2010). Poisoning of dogs with tremorgenic Penicillium toxins. Med Mycol 48(1):188-196.

7. Fernandez, A.L., Lee JA, Rahilly L, Hovda L, Brutlag AG, Engebretsen K. (2011). The use of intravenous lipid emulsion as an antidote in veterinary toxicology. J Vet EmergCrit Care. 21: 309-320. DOI: http://dx.doi.org/10.1111/j.1476-4431.2011.00657.x

8. Fettiplace MR, Weinberg G. Past, present and future of lipid resuscitation therapy. J Parenter Enteral Nutr 2015; 39(1 Suppl):72S-83S. DOI: http://dx.doi.org/10.1177/0148607115595979

9. French $D$, et al. Partition constant and volume of distribution as predictors of clinical efficacy of lipid rescue for toxicological emergencies. Clinical Toxicology (2011), Early Online, 1-9.

DOI: http://dx.doi.org/10.3109/15563650.2011.617308

10. Gwaltney-Brant, S. and Meadows, I. (2012) Use of Intravenous lipid emulsions for treating certain poisoning cases in small animals. Veterinary Clinics of North America: Small Animal Practice 42, 251262. DOI: http://dx.doi.org/10.1016/i.cvsm.2011.12.001

11. Haworth, M.D. and Smart, L. (2012) Use of intravenous lipid therapy in three cases of feline permethrin toxicosis. Journal of Veterinary Emergency Critical Care 22, 697-702.

DOI: http://dx.doi.org/10.1111/j.1476-4431.2012.00804.x

12. Kirsten E. Waratuke, DVM ASPCA Animal Poison Control Center, What's the Take-Home? / NAVC 
Clinician's Brief / October 2012. Pp:49-50.

13. Kuo, K. and Odunayo, A. (2013) Adjunctive therapy with intravenous lipid emulsion and methocarbamol for permethrin toxicity in cats. Journal of Veterinary Emergency Critical Care 23, 436441. DOI: http://dx.doi.org/10.1111/vec.12070

14. Levine, M. et al. (2016) "Systematic review of the effect of intravenous lipid emulsion therapy for nonlocal anesthetics toxicity", Clinical Toxicology, 54(3), pp. 194-221.

DOI: http://dx.doi.org/10.3109/15563650.2015.1126286

15. Norris PJ, Smith CCT, De Belleroche J, et al. Actions of tremorgenic fungal toxins on neurotransmitter release. J Neurochem 1980;34:33-42. 10. DOI: http://dx.doi.org/10.1111/i.1471-4159.1980.tb04618.x

16. Parratt C. (2014) Intravenous lipid rescue for tremorgenic mycotoxicosis: assessment of response to therapy using a smart phone [abstract]. $13^{\text {th }}$ EVECCS Annual Meeting, 12-15 June, Prague, pp272-273.

17. Peacock, R. E., Hosgood, G., Swindells, K. L. and Smart, L. (2015), A randomized, controlled clinical trial of intravenous lipid emulsion as an adjunctive treatment for permethrin toxicosis in cats. Journal of Veterinary Emergency and Critical Care, 25: 597-605. doi:10.1111/vec.12322.

18. Puschner B (2009) Penitrem A and roquefortine. In: Plumlee KH, ed. Clinical Veterinary Toxicology. St. Louis, MO: Mosby. pp:258-259.

19. Rothschild L et al (2010) Intravenous lipid emulsion in Clinical Toxicology Scand J Trauma ResuscEmerg Med. 2010; 18: 51.

20. Rundberget $T$, Wilkins AL (2002) Determination of Penicillium mycotoxins in foods and feeds using liquid chromatography-mass spectromectry. J Chromatog A 2002;964;189-197.

21. Schell MM: Tremorgenic Mycotoxin Intoxication. VET MED 95(4):283-286, 2000.

22. Seitz MA, Burkitt-Creedon JM. Persistent gross lipemia and suspected corneal lipidosis following intravenous lipid therapy in a cat with permethrin toxicosis. J Vet EmergCrit Care 2016 Nov-

Dec;26(6):804-8. DOI: http://dx.doi.org/10.1111/vec.12440

23. Talcott, P. (2013) "Mycotoxins", Small Animal Toxicology, pp. 677-682.

DOI: http://dx.doi.org/10.1016/B978-1-4557-0717-1.00063-6

24. Tiwary $A K$ et al (2009) Using roquefortine $C$ as a biomarker for penitrem $A$ intoxication. J Vet Diagn Invest 21(2):237-239. DOI: http://dx.doi.org/10.1177/104063870902100210

25. Veterinary Poisons Information Service (VPIS) [Online] available from: https://vpisglobal.com/ [Accessed: 22 ${ }^{\text {nd }}$ May 2018]

26. Young KL et al (2003) Tremorgenic mycotoxin intoxication with penitrem $A$ and roquefortine in two dogs. J Am Vet Med Assoc 222(1):52-53. DOI: http://dx.doi.org/10.2460/javma.2003.222.52 


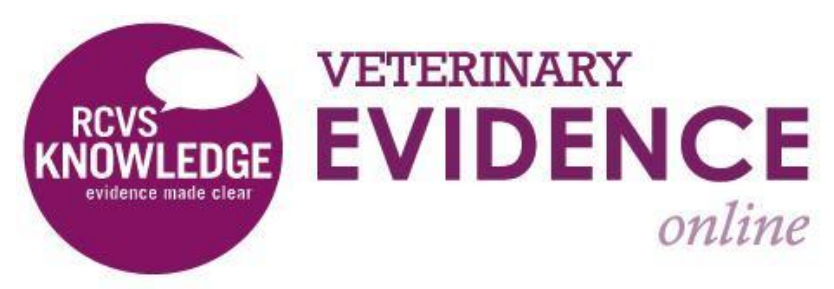

\begin{abstract}
Intellectual Property Rights
Authors of Articles submitted to RCVS Knowledge for publication will retain copyright

in their work, and will be required to grant to RCVS Knowledge a non-exclusive license of the rights of copyright in the materials including but not limited to the right to publish, re-publish, transmit, sell, distribute and otherwise use the materials in all languages and all media throughout the world, and to license or permit others to do
\end{abstract}

so.

Veterinary Evidence and EBVM Network are RCVS Knowledge initiatives. For more information please contact us at editor@veterinaryevidence.org.

RCVS Knowledge is the independent charity associated with the Royal College of Veterinary Surgeons (RCVS). Our ambition is to become a global intermediary for evidence based veterinary knowledge by providing access to information that is of immediate value to practicing veterinary professionals and directly contributes to evidence based clinical decision-making.

\title{
www.veterinaryevidence.org
}

RCVS Knowledge is a registered Charity No. 230886. Registered as a Company limited by guarantee in England and Wales No. 598443.

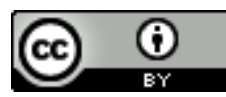

This work is licensed under a Creative Commons Attribution 4.0 International License. 